Pacific Journal of Mathematics

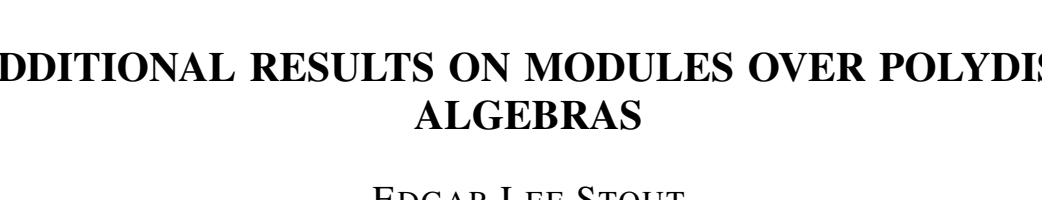




\title{
ADDITIONAL RESULTS ON MODULES OVER POLYDISC ALGEBRAS
}

\author{
E. L. Stout
}

This paper deals with a class $\mathscr{K}_{N}$ of domains in Stein manifolds and with certain algebras of holomorphic functions naturally associated with them.

The class $\mathscr{K}_{N}$ consists of those relatively compact domains $\Delta$ in $N$-dimensional Stein manifolds such that for some neighborhood $\Omega$ of $\bar{\Delta}$ and some neighborhood $W$ of $\bar{U}^{N}$, the closure of $U^{N}=\left\{\left(z_{1}, \cdots, z_{N}\right) \in C^{N}:\left|z_{1}\right|, \cdots,\left|z_{N}\right|<1\right\}$, the unit polydisc in $C^{N}$, there exists a proper holomorphic map $\Phi: \Omega \rightarrow W$ which is nonsingular at every point of $\Phi^{-1}\left(T^{N}\right), T^{N}$ the distinguished boundary of $U^{N}$, and which has, in addition, the property that $\Delta=\Phi^{-1}\left(U^{N}\right)$. The collection of all such maps $\Phi$ is denoted by $\mathscr{U}\left(\Delta, U^{N}\right)$, and if $\Delta, \Delta^{\prime} \in \mathscr{K}_{N}, \mathscr{C}\left(\Delta, \Delta^{\prime}\right)$ denotes the set of all maps $\Psi: \Delta \rightarrow \Delta^{\prime}$ such that if $\Phi \in \mathscr{L}\left(\Delta^{\prime}, U^{N}\right)$, then $\Phi \circ \Psi \in \mathscr{C}\left(\Delta, U^{N}\right)$. For $\Delta \in \mathscr{C}_{N}$ let $\mathscr{A}(\Delta)=\{f \in \mathscr{C}(\bar{\Delta}): f$ is holomorphic in $\Delta\}$, and let $H^{\infty}(\Delta)=\{f: \quad f$ is holomorphic and bounded in $\Delta\}$. If $\Phi \in \mathscr{C}\left(\Delta, \Delta^{\prime}\right)$, then $\mathscr{A}(\Delta)$ is a module over its subalgebra $\Phi^{*} \mathscr{A}\left(\Delta^{\prime}\right)=\left\{f \circ \Phi: \quad f \in \mathscr{A}\left(\Delta^{\prime}\right)\right\}$, and this paper treats the structure of $\mathscr{A}(\Delta)$ as a $\Phi^{*} \mathscr{A}\left(\Delta^{\prime}\right)$-module. The first section of the paper presents an example to show that $\mathscr{A}(\Delta)$ need not be free over $\Phi^{*} \mathscr{A}\left(\Delta^{\prime}\right)$, and the second section shows that it is a finitely generated, projective $\Phi^{*} \mathscr{A}\left(\Delta^{\prime}\right)$ module. The final section establishes certain conditions sufficient for the freeness of $\mathscr{A}(\Delta)$. Parallel results obtain for $H^{\infty}(\Delta)$ as a $\Phi H^{\infty}\left(\Delta^{\prime}\right)$-module.

These results supplement results obtained in [7]. In that paper some of these questions were treated for the special case that $\Delta=U^{N}$. For example, it was shown there that if $\Phi \in \mathscr{C}\left(\Delta, U^{N}\right)$, then $\mathscr{A}(\Delta)$ is a free module over $\Phi^{*} \mathscr{A}\left(U^{N}\right)$. We refer to this paper for some of the elementary properties of the elements of $\mathscr{K}_{N}$ and of $\mathscr{C}\left(\Delta^{\prime}, \Delta\right)$.

Given $\Delta^{\prime}, \Delta \in \mathscr{K}_{N}$, and $\Psi \in \mathscr{C}(\Delta ; \Delta)$, the triple $\left(\Delta ; \Psi \mid \Delta^{\prime}, \Delta\right)$ is an analytic cover in the sense of [5] and consequently has a well defined multiplicity $\lambda: \lambda$ is that integer such that for all points $z \in \Delta^{\prime}$ off a variety, the set $\Psi^{-1}(z)$ consists of $\lambda$ points.

If $M$ is a complex manifold, $\mathscr{S}$ a sheaf on $M$, and $z$ a point of $M$, then $\mathscr{S}_{z}$ denotes the stalk of $\mathscr{S}$ at $z$ and $\mathscr{O}_{M}$ denotes the sheaf of germs of functions holomorphic on $M$. We will usually write $\mathscr{O}_{z}$ instead of $\left(\mathscr{O}_{M}\right)_{3}$. If $K$ is a subset of $M, \mathscr{O}(K)$ denotes the sections of $\mathscr{O}_{M}$ over $K$.

1. An example. If $\Delta, \Delta^{\prime} \in \mathscr{K}_{1}$ and $\Psi \in \mathscr{C}\left(\Delta^{\prime}, \Delta\right)$, then [1] $\mathscr{A}\left(\Delta^{\prime}\right)$ 
is a free module over $\Psi^{*} \mathscr{A}(\Delta)$ whose rank is the multiplicity of $\Psi$. In higher dimensions the analogous result is not true as the following example shows.

We will show that for $N=4,5,6, \cdots$, there exist $\Delta, \Delta^{\prime} \in \mathscr{K}_{N}$ and $\Psi \in \mathscr{M}\left(\Delta^{\prime}, \Delta\right)$ such that $\mathscr{A}\left(\Delta^{\prime}\right)$ admits no set of generators over $\Psi^{*} \mathscr{A}(\Delta)$ consisting of $\lambda$ elements, $\lambda$ the multiplicity of $\Psi$. In our example $\Psi$ will be two-to-one and a local homeomorphism at each point of $\Delta$. Denote by $P_{N}(C)$ and $P_{N}(R)$ respectively $N$-dimensional complex and real projective space. In $P_{N}(C), N \geqq 4$, consider the manifold $V$ consisting of those points with homogeneous coordinates $\left(z_{0}, \cdots, z_{N}\right)$ such that $z_{0}^{2}+\cdots+z_{N}^{2} \neq 0$. In the case $N=2$, this manifold was considered in another connection by Forster [3]. The manifold $V$ is connected since it is the complement in $P_{N}(C)$ of a variety, and, as Forster remarked, it is a Stein manifold. The space $P_{N}(R)$ is contained in a natural way in $V: P_{N}(R)$ is the set of all points which admit real homogeneous coordinates, and, moreover, $P_{N}(R)$ is a deformation retract of $V$. This was the fact which made $V$ useful for Forster, and it is the essential point in the present example. A deformation of $V$ onto $P_{N}(R)$ can be given explicitly as follows [3]. If $z \in V$, let $\left(z_{0}, \cdots, z_{N}\right)$ be homogeneous coordinates for $z$ such that $z_{0}^{2}+\cdots+z_{N}^{2}>0$. Given $t \in[0,1]$, define $H_{t}(z)$ to be the point with homogeneous coordinates $\left(x_{0}+i t y_{0}, \cdots, x_{N}+i t y_{N}\right)$ if $z_{j}=x_{j}+i y_{j}$. Thus, $V$ and $P_{N}(R)$ are of the same homotopy type and in particular they have the same fundamental group, $Z_{2}^{1}$. Consequently, if $\tilde{V}$ denotes the universal covering manifold of $V$ and $\eta: \quad \widetilde{V} \rightarrow V$ the natural projection, then $\eta$ is a local homeomorphism and each fiber $\eta^{-1}(p)$ consists of exactly two points. The manifold $\widetilde{V}$ admits a complex structure with respect to which $\eta$ is a holomorphic map, and when $\tilde{V}$ is endowed with this complex structure, it becomes a Stein manifold. (For the fact that $\widetilde{V}$ is Stein, see [8]).

We set $S=\eta^{-1}\left(P_{N}(R)\right)$, and we shall show that $S$ is, topologically, the $N$-sphere. Since $\eta$ is a local homeomorphism, $S$ is evidently an $N$-dimensional manifold. A priori it is not clear that $S$ is connected; let $S_{0}$ be a component of $S$. Then $\eta$ carries $S_{0}$ onto $P_{N}(R)$, and with the projection $\eta, S_{0}$ is a covering space of $P_{N}(R)$. Let $p_{0} \in P_{N}(R)$ and let $s_{0} \in \eta^{-1}\left(p_{0}\right) \cap S_{0}$. Let $i: \quad\left(P_{N}(R), p_{0}\right) \rightarrow\left(V, p_{0}\right)$ and $j:\left(S_{0}, s_{0}\right) \rightarrow\left(\widetilde{V}, s_{0}\right)$ be inclusion maps. We then have induced homomorphisms of the fundamental groups

$$
\begin{aligned}
i_{*}: & \pi_{1}\left(P_{N}(R), p_{0}\right) \rightarrow \pi_{1}\left(V, p_{0}\right), \\
j_{*}: & \pi_{1}\left(S_{0}, s_{0}\right) \rightarrow \pi_{1}\left(\widetilde{V}, s_{0}\right), \\
\eta_{*}: & \pi_{1}\left(\widetilde{V}, s_{0}\right) \rightarrow \pi_{1}\left(V, p_{0}\right), \\
\left(\eta \mid S_{0}\right)_{*}: & \pi_{1}\left(S_{0}, s_{0}\right) \rightarrow \pi_{1}\left(P_{N}(R), p_{0}\right) .
\end{aligned}
$$

1 The integers $\bmod 2$. 
There is the commutativity relation $\eta_{*} j_{*}=i_{*}\left(\eta \mid S_{0}\right)_{*}$. Since $\tilde{V}$ is the universal covering space of $V, \eta_{*}=0$, and since $P_{N}(R)$ is a deformation retract of $V, i_{*}$ is an isomorphism. Consequently $\left(\eta \mid S_{0}\right)_{*}=0$. From the uniqueness of covering spaces corresponding to a given subgroup of the fundamental group (see, e.g. [6, Th. 6.6.16]) it follows that $S_{0}$ is homeomorphic to the universal covering space of $P_{N}(R)$, i.e., to the $N$-sphere and that $\eta \mid S_{0}$ is two-to-one. Since $\eta$ is two-to-one on $\tilde{V}$, we have that $S=S_{0}$ and consequently that $S$ is an $N$-sphere.

Next we show the existence of a $\Delta \in \mathscr{K}_{N}$ which contains $P_{N}(R)$ and which is contained in $V$. For this purpose, define a map $\Phi: \quad V \rightarrow C^{N}$ by setting

$$
\Phi(z)=\left(\frac{z_{1}^{2}}{z_{0}^{2}+\cdots+z_{N}^{2}}, \cdots, \frac{z_{N}^{2}}{z_{0}^{2}+\cdots+z_{N}^{2}}\right)
$$

if $z \in V$ has homogeneous coordinates $\left(z_{0}, \cdots, z_{N}\right)$. The $\Phi$ so defined is proper. If not, there is a sequence $\left\{\zeta^{(k)}\right\}_{k=1}^{\infty}$ in $C^{N}$ which converges to $\zeta^{(0)} \in C^{N}$ such that for some choice of points $z_{k} \in \Phi^{-1}\left(\zeta^{(k)}\right), \quad z_{k} \rightarrow$ $z_{0} \in P_{N}(C) \backslash V$. Let $z_{0}$ have homogeneous coordinates $\left(z_{0}^{(0)}, \cdots, z_{N}^{(0)}\right)$. Then $\Sigma\left(z_{j}^{(0)}\right)^{2}=0$. We can choose homogeneous coordinates $\left(z_{0}^{(k)}, \cdots, z_{N}^{(k)}\right)$ for $z_{k}$ so that for fixed $j, 0 \leqq j \leqq N, z_{j}^{(k)} \rightarrow z_{j}^{(0)}$. Let $\zeta^{(0)}=\left(\zeta_{1}^{(0)}, \cdots, \zeta_{N}^{(0)}\right)$. Since $\Phi\left(z_{k}\right) \rightarrow \zeta^{(0)}$, we have, for $1 \leqq j \leqq N$,

$$
\zeta_{j}^{(0)}=\lim _{k \rightarrow \infty}\left(z_{j}^{(k)}\right)^{2}\left(\left(z_{0}^{(k)}\right)^{2}+\cdots+\left(z_{0}^{(k)}\right)^{2}\right)^{-1},
$$

and since $z_{k} \rightarrow z_{0}$ and $z_{0} \notin V$, this implies that $z_{j}^{(k)} \rightarrow 0$. From $z_{j}^{(k)} \rightarrow z_{j}^{(0)}$, we conclude that $z_{j}^{(0)}=0$ for $1 \leqq j \leqq N$. The fact that $\Sigma\left(z_{j}^{(0)}\right)^{2}=0$ implies that $z_{0}^{(0)}=0$, so $\left(z_{0}^{(0)}, \cdots, z_{N}^{(0)}\right)=(0, \cdots, 0)$ which is impossible. Thus $\Phi$ is proper. A short calculation shows that with the exception of the points in the variety

$$
E=\left\{\left(\zeta_{1}, \cdots, \zeta_{N}\right) \in C^{N}: \zeta_{1} \cdots \zeta_{N}=0\right\},
$$

every point of $C^{N}$ has exactly $2^{N}$ preimages under $\Phi$ so the multiplicity of $\Phi$ is $2^{N}$. This remark also indicates that $\Phi$ is regular at each point of the sets $\Phi^{-1}\left(\left\{\left(\zeta_{1}, \cdots, \zeta_{N}\right):\left|\zeta_{1}\right|=\cdots=\left|\zeta_{N}\right|=R\right\}\right)$. If $z \in V$ has real homogeneous coordinates, the definition of $\Phi(z)$ shows that $\Phi(z)$ lies in $\bar{U}^{N}$, so if $\varepsilon>0$, then the set

$$
\Delta_{\varepsilon}=\Phi^{-1}\left\{\left(z_{1}, \cdots, z_{N}\right) \in C^{N}: \quad\left|z_{j}\right|<1+\varepsilon \text { for } j=1, \cdots, N\right\}
$$

is an element of $\mathscr{K}_{N}$ with the desired property.

Let $P_{N}(R) \subset \Delta \subset V, \Delta \in \mathscr{K}_{N}$, and let $\Delta^{\prime}=\eta^{-1}(\Delta)$. The mapping $\eta$ : $\widetilde{V} \rightarrow V$ is a covering map and so is certainly an element of $\mathscr{C}\left(\Delta^{\prime}, \Delta\right)$. Assume that $\mathscr{A}\left(\Delta^{\prime}\right)$ is generated as a module over $\eta^{*} \mathscr{A}(\Delta)$ by two elements, $F_{1}$ and $F_{2}$, so that if $f \in \mathscr{A}\left(\Delta^{\prime}\right)$ then for some $g_{1}, g_{2} \in \mathscr{A}(\Delta)$ 
we have that $f(\xi)=F_{1}(z) g_{1}(\eta(z))+F_{2}(\xi) g_{2}(\eta(z))$. In particular, the functions $F_{1}$ and $F_{2}$ must separate points on each of the fibers $\eta^{-1}(z)$, $z \in P_{N}(R)$. Let $S^{N}$ be the standard $N$-sphere in $R^{N+1}$ and denote by $\xi: S^{N} \rightarrow P_{N}(R)$ the usual covering map which identifies antipodal points. If $\tau: S^{N} \rightarrow S$ is a homeomorphism such that $\xi=\eta \circ \tau$, then the mapping $S^{N} \rightarrow C^{2}$ given by $z \rightarrow\left(F_{1}(\tau(z)), F_{2}(\tau(z))\right)$ is continuous and separates antipodal points. Since $C^{2}$ is, topologically, $R^{4}$, and since we have assumed $N \geqq 4$, we have obtained a contradiction to the Borsuk-Ulam Theorem [6, Corollary 4.3.7]. Consequently, $\mathscr{A}\left(\Delta^{\prime}\right)$ is not generated by two elements over $\eta^{*} \mathscr{A}(\Delta)$. This argument shows, in fact, that any set of generators for $\mathscr{A}\left(\Delta^{\prime}\right)$ must contain more than $N / 2$ generators.

2. $\mathscr{A}\left(\Delta^{\prime}\right)$ as a module over $\Psi^{*} \mathscr{A}(\Delta)$. Complementing the previous example, we have the following result.

Theorem 2.1. If $\Delta^{\prime}, \Delta \in \mathscr{K}_{N}$ and $\Psi \in \mathscr{M}\left(\Delta^{\prime}, \Delta\right)$, then $\mathscr{A}\left(\Delta^{\prime}\right)$ and $H^{\infty}\left(\Delta^{\prime}\right)$ are finitely generated projective modules over $\Psi^{*} \mathscr{A}(\Delta)$ and $\Psi^{*} H^{\infty}(\Delta)$ respectively.

Proof. It is easy to see that $\mathscr{A}\left(\Delta^{\prime}\right)$ is finitely generated over $\Psi^{*} \mathscr{A}(\Delta)$ and that a similar result obtains concerning $H^{\infty}\left(\Delta^{\prime}\right)$. Let $\Psi \in \mathscr{M}\left(\Delta, U^{N}\right)$. Then $\Phi \circ \Psi \in \mathscr{C}\left(\Delta^{\prime}, U^{N}\right)$, and consequently $\mathscr{A}\left(\Delta^{\prime}\right)$ is finitely generated over $(\Phi \circ \Psi)^{*} \mathscr{A}\left(U^{N}\right)$ : We know by [7, Th. I. 4] that for some $B_{1}, \cdots, B_{m} \in \mathcal{O}\left(\bar{\Delta}^{\prime}\right)$, each $f \in \mathscr{A}(\Delta)$ is of the form

$$
f=\sum B_{j} f_{j} \circ \Phi \circ \Psi
$$

for some choice of $f_{j}$ in $\mathscr{A}\left(U^{v}\right)$. Since $f \circ \Phi$ is in $\mathscr{A}(\Delta)$, this shows that $\mathscr{A}\left(\Delta^{\prime}\right)$ is finitely generated over $\Psi^{*} \mathscr{A}(\Delta)$. The case of $H^{\infty}\left(\Delta^{\prime}\right)$ can be treated in the same way. Somewhat more is required to show that these modules are projective.

Since $\Psi \in \mathscr{C}\left(\Delta^{\prime}, \Delta\right)$, there is a neighborhood $\Omega^{\prime}$ of $\overline{\Delta^{\prime}}$ which is mapped properly onto a neighborhood $\Omega$ of $\bar{\Delta}$ by $\Psi$. We know [7, Lemma 1.2] that the direct image sheaf $\Psi * \mathcal{O}_{\Omega^{\prime}}$ is locally free of rank $\lambda, \lambda$ the multiplicity of $\Psi$ on $\Omega$. Let $W$ be a relatively compact open set which is a Stein manifold and which satisfies $\Omega \supset \bar{W} \supset W \supset \bar{\Delta}$. By Cartan's Theorem A and compactness, there exist

$$
\widetilde{F}_{1}, \cdots, \widetilde{F}_{q} \in \Gamma\left(\Omega, \Psi * \mathcal{O}_{\Omega}\right)
$$

such that if $z \in \bar{W}$, then the germs $\left(\widetilde{F}_{j}\right)_{z}$ generate $\left(\Psi * \mathcal{O}_{\Omega^{\prime}}\right)_{z}{ }^{2}$. Thus, if $H$ is the sheaf homomorphism $\mathcal{O}^{q} \rightarrow \Psi_{*} \mathcal{O}_{a^{\prime}}$ given by $H\left(\boldsymbol{f}_{1}, \cdots, \boldsymbol{f}_{q}\right)=$ $\sum \boldsymbol{f}_{j}\left(\widetilde{F}_{j}\right)_{z}$ for all $\boldsymbol{f}_{j} \in \mathcal{O}_{z}$ then

\footnotetext{
${ }^{2}$ By a result of Forster and Ramspott [4, Satz 2], we can take $q=\lambda+[N / 2]$.
} 


$$
\mathrm{O}^{q} \stackrel{H}{\longrightarrow} \Psi_{*} \mathrm{O}_{\Omega^{\prime}} \longrightarrow 0
$$

is exact over $W$.

We now need a lemma which is surely well known but for which we are unable to provide a reference. (Remarks of the referee have enabled us to abbreviate our discussion of this lemma).

LEMma 2.2. If $\mathscr{S}_{1} \stackrel{H}{\longrightarrow} \mathscr{S}_{2} \rightarrow 0$ is an exact sequence of locally free sheaves over a complex manifold $M$, then ker $H$ is locally free.

Proof. The sheaves $\mathscr{S}_{1}$ and $\mathscr{S}_{2}$ are locally free and the question is local, so we can suppose $\mathscr{S}_{1}=\mathcal{O}^{p}, \mathscr{S}_{2}=\mathcal{O}^{q}$. Since $\operatorname{ker} H$ is coherent, it suffices to prove that for each $z \in M$, the stalk (ker $H)_{z}$ is a free $O_{z}$-module. (See Lemma 1.2 of [7]). We have the exact sequence

$$
0 \longrightarrow \operatorname{ker} H \longrightarrow \mathcal{O}^{p} \longrightarrow \mathrm{O}^{q} \longrightarrow 0
$$

so we can invoke [2, I. 2.5, Proposition 5 and II. 5.2, Corollary 2] to conclude that $(\operatorname{ker} H)_{z}$ is a projective $O_{z}$-module. Since projective modules over local rings are free, we can conclude that (ker $H)_{z}$ is free as desired.

To continue with the proof of the theorem, we apply the lemma to the sequence

$$
0 \longrightarrow \operatorname{ker} H \longrightarrow \mathrm{O}^{q} \stackrel{H}{\longrightarrow} \Psi * \mathrm{O}_{\Omega^{\prime}} \longrightarrow 0
$$

over $W$ obtained from (1), and we find that this is an exact sequence of locally free sheaves. Consequently [4, Th. VIII C 7], the sequence (2) splits, and in particular there is a sheaf isomorphism $L$ of $\Psi_{*} \mathcal{O}_{\Omega^{\prime}}$ into $\mathcal{O}^{q}$ such that $H \circ L$ is the identity on $\Psi_{*} \mathcal{O}_{\Omega^{\prime}}$ and $L \circ H$ is a projection of $\mathcal{O}^{q}$ onto the range of $L$. Apply this to the spaces of sections over $\bar{\Delta}$ and pull the resulting statement back to $\bar{\Delta}^{\prime}$ by way of the map $\Psi$. We find that there are exact sequences of $\Psi^{*} \mathcal{O}(\bar{\Delta})$-module homomorphisms

$$
0 \longrightarrow \mathcal{O}\left(\overline{\Delta^{\prime}}\right) \stackrel{L^{\prime}}{\longrightarrow}\left(\Psi^{*} \mathcal{\odot}(\bar{\Delta})\right)^{q}
$$

and

$$
\left(\Psi^{*} \mathscr{O}(\bar{\Delta})\right)^{q} \stackrel{H^{\prime}}{\longrightarrow} \mathcal{O}\left(\bar{\Delta}^{\prime}\right) \longrightarrow 0 \text {. }
$$

Here $H^{\prime}\left(f_{1} \circ \Psi, \cdots, f_{q} \circ \Psi\right)=\sum F_{j} f_{j} \circ \Psi$ where $F_{j} \in \mathcal{O}\left(\overline{\Delta^{\prime}}\right)$ corresponds to the section $\widetilde{F}_{j}$ of $\Psi_{*} \mathcal{O}_{\Omega^{\prime}}, L^{\prime} \circ H^{\prime}$ projects $\Psi^{*} \mathcal{O}(\bar{\Delta})$ onto the range of $L^{\prime}$, and $H^{\prime} \circ L^{\prime}$ is the identity on $\mathscr{O}\left(\bar{\Delta}^{\prime}\right)$. This establishes $\mathscr{O}\left(\overline{\Delta^{\prime}}\right)$ as a finitely generated, projective $\Psi^{*} \mathscr{O}(\bar{\Delta})$-module.

To treat $\mathscr{A}\left(\Delta^{\prime}\right)$ and $H^{\infty}\left(\Delta^{\prime}\right)$, note that since $L$ is a sheaf isomorphism, $L^{\prime}$ extents to an isomorphism $L^{\prime \prime}$ of $\mathscr{O}\left(\Delta^{\prime}\right)$ into $\left(\Psi^{*} \mathscr{O}(\Delta)\right)^{q}$ and 
that $H^{\prime}$ extends to a homomorphism $H^{\prime \prime}$ of $\left(\Psi^{*} \mathscr{O}(\Delta)\right)^{q}$ to $\mathscr{O}\left(\Delta^{\prime}\right)$. The form of $H$ shows that $H^{\prime \prime}$ carries $\left(\Psi^{*} \mathscr{A}(\Delta)\right)^{q}$ into $\mathscr{A}\left(\Delta^{\prime}\right)$ and $\left(\Psi^{*} H^{\infty}(\Delta)\right)^{q}$ into $H^{\infty}\left(\Delta^{\prime}\right)$. In fact $H^{\prime \prime}$ carries $\left(\Psi^{*} \mathscr{A}(\Delta)\right)^{q}$ and $\left(\Psi^{*} H^{\infty}(\Delta)\right)^{q}$ onto $\mathscr{A}\left(\Delta^{\prime}\right)$ and $H^{\infty}\left(\Delta^{\prime}\right)$ respectively. As we noted at the beginning of the proof, if $f \in \mathscr{A}\left(\Delta^{\prime}\right)$, then $f=\sum B_{j} f_{j} \circ \Psi$ for some $f_{j} \in \mathscr{A}(\Delta)$ and some $B_{j} \in \mathcal{O}\left(\bar{\Delta}^{\prime}\right)$. We have $B_{j}=H^{\prime}\left(\widetilde{B}_{j}\right)$ for some $\widetilde{B}_{j} \in\left(\Psi^{*} \mathcal{O}(\bar{\Delta})\right)^{q}$. On $\left(\Psi^{*} \mathscr{A}(\Delta)\right)^{q}, H^{\prime \prime}$ acts as a $\Psi^{*} \mathscr{A}(\Delta)$-module homomorphism, so we have $f=H^{\prime \prime}\left(\sum \widetilde{B}_{j} f_{j} \circ \Psi\right)$. The case that $f$ lies in $H^{\infty}\left(\Delta^{\prime}\right)$ may be treated in a similar way.

Also, $L^{\prime \prime}$ carries $\mathscr{A}\left(\Delta^{\prime}\right)$ into $\left(\Psi^{*} \mathscr{A}(\Delta)\right)^{q}$ and $H^{\infty}\left(\Delta^{\prime}\right)$ into $\left(\Psi^{*} H^{\infty}(\Delta)\right)^{q}$. If $f \in \mathscr{A}\left(\Delta^{\prime}\right)$, we write $f=\sum B_{j} f_{j} \circ \Psi$ as above. Then $L^{\prime \prime} f=\sum f_{j} \circ L^{\prime \prime} B_{j}$. We have $L^{\prime \prime} B_{j} \in\left(\Psi^{*} \mathscr{O}(\bar{\Delta})\right)^{q} \subset\left(\Psi^{*} \mathscr{A}(\Delta)\right)^{q}$. Thus $L^{\prime \prime} f$ is in $\left(\Psi^{*} \mathscr{A}(\Delta)\right)^{q}$ as asserted. The $H^{\infty}$ case follows in the same way.

The operator $L^{\prime \prime} \circ H^{\prime \prime}$ acts on $\left(\Psi^{*} \mathscr{A}(\Delta)\right)^{q}$ as a projection with range the range of $L^{\prime \prime}$ on $\mathscr{A}\left(\Delta^{\prime}\right)$. To see this, note first that the range of $L^{\prime \prime} \circ H^{\prime \prime}$ is $L^{\prime \prime}\left(\mathscr{A}\left(\Delta^{\prime}\right)\right)$, for $H^{\prime \prime}$ carries $\left(\Psi^{*} \mathscr{A}(\Delta)\right)^{q}$ onto $\mathscr{A}\left(\Delta^{\prime}\right)$. If $f \in \mathscr{A}\left(\Delta^{\prime}\right)$, then since $H^{\prime \prime} \circ L^{\prime \prime}$ is the identity, we find that $L^{\prime \prime} \circ H^{\prime \prime}\left(L^{\prime \prime} f\right)=L^{\prime \prime} f$, so $L^{\prime \prime} \circ H^{\prime \prime}$ is a projection. Since $L^{\prime \prime}$ takes $\mathscr{A}\left(\Delta^{\prime}\right)$ isomorphically into $\left(\Psi^{*} \mathscr{A}(\Delta)\right)^{q}$, we have proved that $\mathscr{A}\left(\Delta^{\prime}\right)$ is a projective $\Psi^{*} \mathscr{A}(\Delta)$-module. In the same way, it follows that $H^{\infty}\left(\Delta^{\prime}\right)$ is a projective $\Psi^{*} H^{\infty}(\Delta)$-module, and the proof of the theorem is concluded.

3. Criteria for the freeness of $\mathscr{A}\left(\Delta^{\prime}\right)$ over $\Psi^{*} \mathscr{A}(\Delta)$. There are certain cases in which $\mathscr{A}\left(\Delta^{\prime}\right)$ is necessarily free over $\Psi^{*} \mathscr{A}(\Delta)$. To introduce some of these we need to consider products. Suppose $\Delta_{1} \in \mathscr{K}_{N_{1}}, \Delta_{2} \in \mathscr{K}_{N_{2}}$, and let $\Phi_{j} \in \mathscr{C}\left(\Delta_{j}, U^{N_{j}}\right)$. If $\Omega_{j}$ is a neighborhood of $\bar{J}_{j}$ which is mapped properly into the neighborhood $W_{j}$ of $\bar{U}^{N_{j}}$, $j=1,2$, then the map $\Psi: \Omega_{1} \times \Omega_{2} \rightarrow W_{1} \times W_{2}$ given by

$$
\Psi\left(z_{1}, z_{2}\right)=\left(\Phi_{1}\left(z_{1}\right), \Phi_{2}\left(z_{2}\right)\right) \in W_{1} \times W_{2}
$$

is proper, and $A_{1} \times \Delta_{2}=\Psi^{-1}\left(U^{N 1+N 2}\right)$. Moreover, $\Psi$ is a local homeomorphism at each point of $\Psi^{-1}\left(T^{N 1+N 2}\right)$. Thus $\Delta_{1} \times \Delta_{2} \in \mathscr{K}_{N 1+N 2}$, and $\Psi \in \mathscr{C l}\left(\Delta_{1} \times \Delta_{2}, U^{N 1+N 2}\right)$. Similarly, if we are given $\Delta_{1}^{\prime}$ and $\Delta_{2}^{\prime}$ in $\mathscr{K}_{N 1}$ and $\mathscr{K}_{N 2}$ respectively and if $\Phi_{j} \in \mathscr{M}\left(\Delta_{j}^{\prime}, \Delta_{j}\right), j=1,2$, then the map $\Phi_{1} \times \Phi_{2}$ from $\Delta_{1}^{\prime} \times \Delta_{2}^{\prime}$ to $\Delta_{1} \times \Delta_{2}$ defined by $\Phi_{1} \times \Phi_{2}\left(z_{1}, z_{2}\right)=\left(\Phi_{1}\left(z_{1}\right), \Phi_{2}\left(z_{2}\right)\right)$ is an element of $\mathscr{C}\left(\Delta_{1}^{\prime} \times \Delta_{2}^{\prime}, \Delta_{1} \times \Delta_{2}\right)$. If $\Phi_{j}$ has multiplicity $\lambda_{j}$, then $\Phi_{1} \times \Phi_{2}$ has multiplicity $\lambda_{1} \lambda_{2}$.

TheOREM 3.1. If for $j=1,2, \Delta_{j}, \Delta_{j}^{\prime} \in \mathscr{K}_{N_{j}}$, if $\Phi_{j} \in \mathscr{M}\left(\Delta_{j}^{\prime}, \Delta_{j}\right)$ is of multiplicity $\lambda_{j}$, and if $\mathscr{A}\left(\Delta_{j}^{\prime}\right)$ is free of rank $\lambda_{j}$ over $\Psi^{*} \mathscr{A}\left(\Delta_{j}\right)$, then $\mathscr{A}\left(\Delta_{1}^{\prime} \times \Delta_{1}^{\prime}\right)$ is free of rank $\lambda_{1} \lambda_{2}$ over $\left(\Phi_{1} \times \Phi_{2}\right) * \mathscr{A}\left(\Delta_{1} \times \Delta_{2}\right)$.

Before giving the proof of this theorem, let us mention that by Theorem 2.3 of [7], if $\Phi \in \mathscr{C}\left(U^{N}, U^{N}\right)$, then in an obvious extension 
of the above notation, $\Phi=\varphi_{1} \times \cdots \times \varphi_{N}$ where each $\varphi_{j} \in \mathscr{M}(U, U)$ is a finite Blaschke product.

Proof. Let $\left\{F_{1}^{(1)}, \cdots, F_{\lambda_{1}}^{(1)}\right\}$ be a free basis for $\mathscr{A}\left(\Delta_{1}^{\prime}\right)$ over $\Phi_{1}^{*} \mathscr{A}\left(\Delta_{1}\right)$ and let $\left\{F_{1}^{(2)}, \cdots, F_{\lambda_{2}}^{(2)}\right\}$ be one for $\mathscr{A}\left(\Delta_{2}^{\prime}\right)$ over $\Phi_{2}^{*} \mathscr{A}\left(\Delta_{2}\right)$. We assert that the set $\left\{F_{j}^{(1)} F_{k}^{(2)}: 1 \leqq j \leqq \lambda_{1}, 1 \leqq k \leqq \lambda_{2}\right\}$ is a free basis for $\mathscr{A}\left(\Delta_{1}^{\prime} \times \Delta_{2}^{\prime}\right)$ over $\left(\Phi_{1} \times \Phi_{2}\right)^{*} \mathscr{A}\left(\Delta_{1} \times \Delta_{2}\right)$.

Define $E_{1}(z)$ for $z \in \bar{\Delta}_{1}^{\prime}$ to be the set $\Phi_{1}^{-1}\left(\Phi_{1}(z)\right)$, and define $E_{2}(z)$ for $z \in \overline{\Delta_{2}^{\prime}}$ in an analogous way. In general $E_{1}(z)$ will consist of $\lambda_{1}$ points. Since $\left\{F_{k}^{(1)}\right\}$ is a free basis for $\mathscr{A}\left(\Delta_{1}^{\prime}\right)$ over $\Phi_{1}^{*} \mathscr{A}\left(\Delta_{1}\right)$, each $f \in \mathscr{A}\left(\Delta_{1}^{\prime}\right)$ has a unique expression in the form

$$
f(z)=F_{1}^{(1)}(z) f_{1}\left(\Phi_{1}(z)\right)+\cdots+F_{\lambda_{1}}^{(1)}(z) f_{\lambda_{1}}\left(\Phi_{1}(z)\right)
$$

with $f_{1}, \cdots, f_{\lambda_{1}} \in \mathscr{A}\left(\Delta_{1}\right)$. The functions $f_{j} \circ \Phi_{1}$ can be computed explicitly by Cramer's rule: $f_{j}\left(\Phi_{1}(z)\right)=D_{j}(z) D^{-1}(z)$ where, for $z \in \overline{\Delta^{\prime}}$ such that $E_{1}(z)$ consists of $\lambda_{1}$ distinct points, say $E_{1}(z)=\left\{z_{1}, \cdots, z_{z_{1}}\right\}$, we have

$$
D(z)=\operatorname{det}\left(F_{j}^{(1)}\left(z_{k}\right)\right)_{1 \leqq j, k \leqq \lambda_{1}},
$$

and $D_{j}(z)$ is obtained from $D(z)$ by replacing the $j^{\text {th }}$ column by the column vector $\left(f\left(z_{1}\right), \cdots, f\left(z_{z_{1}}\right)\right)$.

If we are given an element $G \in \mathscr{A}\left(\Delta_{1}^{\prime} \times \Delta_{2}^{\prime}\right)$, then for fixed $w \in \bar{\Delta}_{2}^{\prime}$, the preceding remarks may be applied to the element $G(\cdot, w)$ of $\mathscr{A}\left(\Delta_{1}^{\prime}\right)$ :

$$
G(\mathfrak{z}, w)=\sum_{j=1}^{\lambda_{1}} F_{j}^{(1)}(\xi) f_{j}\left(\Phi_{1}(\xi), w\right)
$$

where, for fixed $w, f_{j}(\cdot, w) \in \mathscr{A}\left(\Delta_{1}\right)$. The expression for $f_{j}$ as a certain quotient of determinants shows that $f_{j}\left(\Phi_{1}(z), w\right)$ is in fact an element of $\mathscr{A}\left(\Delta_{1}^{\prime} \times \Delta_{2}^{\prime}\right)$. Thus, for fixed $z$, we can write

$$
f_{j}\left(\Phi_{1}(z), w\right)=\sum_{k=1}^{\lambda_{2}} F_{k}^{(2)} w g_{j, k}\left(z, \Phi_{2}(w)\right) \text {. }
$$

Again, we can compute the functions $g_{j, k}$ explicitly: If $E_{2}(w)=$ $\left\{w_{1}, \cdots, w_{\lambda_{2}}\right\}$, then $g_{j, k}\left(z, \Phi_{2}(w)\right)=\widetilde{D}_{k}\left(z, \Phi_{2}(w)\right) \widetilde{D}\left(z, \Phi_{2}(w)\right)^{-1}$ where, as before,

$$
\widetilde{D}\left(\mathfrak{z}, \Phi_{2}(w)\right)=\operatorname{det}\left(F_{k}^{(2)}\left(w_{m}\right)\right)_{1 \leqq k, m \leq \lambda_{2}}
$$

and $\widetilde{D}_{k}\left(z, \Phi_{2}(w)\right)$ is obtained by replacing the $k^{\text {th }}$ column of $\widetilde{D}$ by the column vector $\left(f_{j}\left(\Phi_{1}(z), w_{1}\right), \cdots, f_{j}\left(\Phi_{1}(z), w_{\lambda_{2}}\right)\right)$. This representation for $g_{j, k}$ shows that for fixed $w, g_{j, k}\left(z, \Phi_{2}(w)\right)$ is, as a function of $z$, constant on the set $E_{1}(z)$. Thus, we can write $g_{j, k}\left(z, \Phi_{2}(w)\right)=h_{j, k}\left(\Phi_{1}(z), \Phi_{2}(w)\right)$ for some suitably chosen $h_{j, k} \in \mathscr{A}\left(\Delta_{1} \times \Delta_{2}\right)$. We now have the representation

$$
G(\mathfrak{\jmath}, w)=\Sigma F_{j}^{(1)}(\mathfrak{\jmath}) F_{k}^{(2)}(w) h_{j, k}\left(\Phi_{1}(\mathfrak{\jmath}), \Phi_{2}(w)\right)
$$


for $G$. Thus, $\left\{F_{j}^{(1)} F_{k}^{(2)}\right\}$ is a set of generators for $\mathscr{A}\left(\Delta_{1}^{\prime} \times \Delta_{2}^{\prime}\right)$ over $\left(\Phi_{1} \times \Phi_{2}\right)^{*} \mathscr{A}\left(\Delta_{1} \times \Delta_{2}\right)$.

That $F_{j}^{(1)} F_{k}^{(2)}$ are free generators is now clear: If there were a nontrivial relation

$$
0=\Sigma F_{j}^{(1)}(\mathfrak{z}) F_{k}^{(2)}(w) h_{j, k}\left(\Phi_{1}(\mathfrak{z}), \Phi_{2}(w)\right) .
$$

Then for some fixed choice of $w$ we could regard this as a nontrivial relation among the functions $F_{1}^{(1)}, \cdots, F_{\lambda_{1}}^{(1)}$. But since $\left\{F_{j}^{(1)}\right\}$ is a free basis for $\mathscr{A}\left(\Delta_{1}^{\prime}\right)$, no such relation can exist, and the theorem is proved.

It is clear that a similar result obtains for products of more than two elements of $\mathscr{K}_{N}$ and that an analogous theorem holds for bounded functions.

We saw in [7] that if $\Delta \in \mathscr{K}_{N}$ and $\Phi \in \mathscr{M}\left(\Delta, U^{N}\right)$, then $\mathscr{A}(\Delta)$ is a free module over $\Phi^{*} \mathscr{\ell}\left(U^{N}\right)$. The essential ingredient of this proof is the fact that for some neighborhood $\Omega$ of $\bar{\Delta}$ and some neighborhood $W$ of $U^{N}$, the sheaf $\Phi_{*} \mathcal{O}_{\Omega}$ is a free sheaf over $W$. The relation between the freeness of $\mathscr{A}(\Delta)$ over $\Phi^{*} \mathscr{A}\left(U^{N}\right)$ and the freeness of the sheaf $\Phi_{*} \mathcal{O}_{\Omega}$ is one which persists in more general settings.

THEOREM 3.2. If $\Delta, \Delta^{\prime} \in \mathscr{K}_{N}$, if $\Phi \in \mathscr{C}\left(\Delta^{\prime}, \Delta\right)$, and if $\mathscr{A}\left(\Delta^{\prime}\right)$ is free of rank $\lambda, \lambda$ the multiplicity of $\Phi$, over $\Phi^{*} \mathscr{A}(\Delta)$, then for some neighborhood $\Omega$ of $\overline{\bar{\Delta}^{\prime}}$ on which $\Phi$ is defined, the sheaf $\Phi_{*} O_{\Omega}$ is free over $\mathscr{O}_{\Phi(\Omega)}$.

Before proving the theorem, a simple preliminary observation is needed.

Lemma 3.3. If $\Delta, \Delta^{\prime} \in \mathscr{K}_{N}$, if $\Phi \in \mathscr{M}\left(\Delta^{\prime}, \Delta\right)$, and if $\mathscr{A}\left(\Delta^{\prime}\right)$ is free over $\Phi^{*} \mathscr{A}(\Delta)$, then there exists a set of free generators for $\mathscr{A}\left(\Delta^{\prime}\right)$ which consists of functions holomorphic on a neighborhood of $\overline{\bar{J}^{\prime}}$.

Proof. By hypothesis there exists an isomorphism

$$
L: \quad \Phi^{*} \mathscr{A}(\Delta)^{q} \rightarrow \mathscr{A}\left(\Delta^{\prime}\right) ;
$$

it is of the form

$$
L\left(f_{1} \circ \Phi, \cdots, f_{q} \circ \Phi\right)=\sum_{j=1}^{q} F_{j} f_{j} \circ \Phi
$$

for some fixed elements $F_{1}, \cdots, F_{q} \in \mathscr{A}\left(\Delta^{\prime}\right)$. The operator $L$ is continuous and so it has a continuous inverse $L^{-1}$. If $S: \Phi^{*} \mathscr{A}(\Delta)^{q} \rightarrow \mathscr{A}\left(\Delta^{\prime}\right)$ is near $L$ in the norm topology of $\mathscr{L}\left(\Phi^{*} \mathscr{A}(\Delta)^{q}, \mathscr{A}\left(\Delta^{\prime}\right)\right){ }^{3}$ then $S \circ L^{-1}$

${ }^{3}$ We use $\mathscr{C}(X, Y)$ to denote the continuous linear operators from the Banach space $X$ to the Banach space $Y$. 
is near the identity of $\mathscr{L}\left(\mathscr{A}\left(\Delta^{\prime}\right), \mathscr{A}\left(\Delta^{\prime}\right)\right)$ and so is invertible. Thus, if $S$ is near $L, S$ is also an isomorphism. Therefore if we choose functions $G_{1}, \cdots, G_{q}$ holomorphic on a neighborhood of $\overline{\Delta^{\prime}}$ so that $G_{j}$ is uniformly near $F_{j}$ on $\overline{\Delta^{\prime}}$ and if we define

$$
S: \quad \Phi^{*} \mathscr{A}(\Delta)^{q} \rightarrow \mathscr{A}\left(\Delta^{\prime}\right)
$$

by $S\left(f_{1} \circ \Phi, \cdots, f_{q} \circ \Phi\right)=\Sigma G_{j} f_{j} \circ \Phi$, then $S$ is a $\Phi^{*} \mathscr{A}(\Delta)$-module isomorphism, i.e., $\left\{G_{1}, \cdots, G_{q}\right\}$ is a free basis for $\mathscr{A}\left(\Delta^{\prime}\right)$ over $\Phi^{*} \mathscr{A}(\Delta)$. That the desired approximating functions exist is contained in [7, Corollary I. 6].

Proof of Theorem 3.4. Let $F_{1}, \cdots, F_{\lambda} \in \mathcal{O}\left(\bar{\Delta}^{\prime}\right)$ be a free basis for $\mathscr{A}\left(\Delta^{\prime}\right)$ over $\Phi^{*} \mathscr{A}(\Delta)$, and let $\Omega$ be a neighborhood of $\bar{\Delta}$ such that $F_{1}, \cdots, F_{\lambda}$ are all holomorphic in $\Omega^{\prime}=\Phi^{-1}(\Omega)$. We have a homomorphism $\mathscr{F}: \quad \mathcal{O}_{\Omega}^{\lambda} \rightarrow \Phi_{*} \mathcal{O}_{\Omega^{\prime}}$ defined by $\mathscr{F}\left(\boldsymbol{f}_{1}, \cdots, \boldsymbol{f}_{\lambda}\right)=\Sigma\left(\widetilde{F}_{j}\right)_{3} \boldsymbol{f}_{j}$ for all

$$
\left(\boldsymbol{f}_{1}, \cdots, \boldsymbol{f}_{2}\right) \in \mathcal{O}_{z}^{z}, z \in \Omega^{\prime} \text {. }
$$

Here $\widetilde{F}_{j}$ is the section of $\Phi_{*} \mathcal{O}_{a^{\prime}}$ corresponding to $F_{j}$, and $\left(\widetilde{F}_{j}\right)_{z}$ is its germ at $z$. We shall show that $\mathscr{F}$ is a sheaf isomorphism at least when we restrict attention to some, possibly smaller neighborhood of $\bar{\Delta}$.

Consider a point $z \in \bar{\Delta}$. We shall show that the stalk map given by $\mathscr{F}$ is an isomorphism in the stalk over $z$. Since $\Phi_{*} \mathcal{O}_{\Omega}$, is locally free of rank $\lambda$, there is an isomorphism $L: \mathcal{O}_{z}^{\lambda} \rightarrow\left(\Phi_{*} \mathscr{O}_{\Omega^{\prime}}\right)_{3}$. Denote by $\boldsymbol{e}_{j}$ the element $(0, \cdots, 0,1,0, \cdots, 0)\left(1\right.$ in the $j^{\text {th }}$ place $)$ of $\mathcal{O}_{z}^{\lambda}$, and let $\boldsymbol{g}_{j} \in\left(\Phi_{*} \mathscr{O}_{\Omega^{\prime}}\right)_{z}$ be the image of $\boldsymbol{e}_{j}$ under $L$. There is a neighborhood $W$ of $z$ in which all the germs $\boldsymbol{g}_{j}$ can be represented by sections of $\Phi_{*} \mathscr{O}_{\Omega^{\prime} ;}$ call these sections $g_{j}$. Thus, $g_{j} \in \mathcal{O}\left(\Phi^{-1}(W)\right)$. If $V \subset W$ is a small neighborhood of $z$, then on $\left(\Phi^{-1}(V)\right)^{-}$, the functions $g_{j}$ will admit uniform approximation by functions $G_{j} \in \mathcal{O}(\bar{\Delta})$. We can choose the approximating functions $G_{j}$ so that in the expression

$$
G_{j}=\Sigma F_{k} h_{k}^{(j)} \circ \Phi \quad\left(h_{k}^{(j)} \in \mathscr{A}(\Delta)\right)
$$

the functions $h_{k}^{(j)}$ lie in $\mathscr{O}(\bar{\Delta})$. The $G_{j}$ give sections $\widetilde{G}_{j}$ of $\Phi_{*} \mathcal{O}_{a}$, which lie in the range of $\mathscr{F}$. Moreover, for any choice of $G_{j}$, we obtain a homomorphism $L^{\prime}: \quad \mathscr{O}_{z}^{\prime} \rightarrow\left(\Phi_{*} \mathscr{O}_{g^{\prime}}\right)_{z}$ by setting $L^{\prime}\left(\boldsymbol{e}_{j}\right)=\left(\widetilde{G}_{j}\right)_{z}$ If the functions $G_{j}$ approximate the functions $g_{j}$ sufficiently well, the $L^{\prime}$ so obtained will be an isomorphism since $L$ is. Fix a choice of the $G_{j}$ so that $L^{\prime}$ is an isomorphism.

Using $L^{\prime}$, we can see that $\mathscr{F}$ is onto (in the stalk over $z$ ), for the range of $\mathscr{F}$ contains $\left\{\widetilde{G}_{1}, \cdots, \widetilde{G}_{\lambda}\right\}$ and so it contains the module generated by this set. Since $L^{\prime}$ is onto, it follows that this module is the whole of $\left(\Phi_{*} \mathcal{O}_{a}\right)_{3}$.

The fact that $\mathscr{F}$ is one-to-one in the stalk over $z$ follows from 
Cartan's Theorem A. If $\mathscr{F}\left(f_{1}, \cdots, f_{\lambda}\right)=0,\left(f_{1}, \cdots, f_{\lambda}\right) \in \mathcal{O}_{z}^{\lambda}$, then $\mathscr{R}$, the sheaf of relations among $\widetilde{F}_{1}, \cdots, \widetilde{F}_{\lambda}$ is nontrivial over $\bar{\Delta}$. Thus by Cartan's Theorem A, there is a nontrivial section of $\mathscr{K}$ over $\bar{A}$, i.e., there exist $h_{1}, \cdots, h_{\lambda} \in \mathcal{O}(\bar{\Delta})$ not all of which are the zero function, such that $\Sigma h_{j} \widetilde{F}_{j}=0$, i.e., $\Sigma F_{j} h_{j} \circ \Phi=0$. This is impossible since $\left\{F_{1}, \cdots, F_{\lambda}\right\}$ is a free basis for $\mathscr{A}\left(\Delta^{\prime}\right)$ over $\Phi^{*} \mathscr{A}(\Delta)$.

Thus, for all $z \in \Delta, \mathscr{F}$ carries $\mathcal{O}_{z}^{\lambda}$ isomorphically onto $\left(\Phi^{*} \mathscr{O}_{\Omega^{\prime}}\right)_{z}$. Consequently, the same assertion holds for all $z$ in a neighborhood of $\bar{A}$, and the theorem is proved.

Note added in proof. My colleague S.J. Sidney has observed that with a somewhat more careful use of the Borsuk-Ulam theorem, it is possible to show that the example of Section I is valid in dimensions two and three as well as in higher dimensions. Consider, in the notation of that section, a $\Delta \in \mathscr{K}_{N}, N \geqq 2$ with $P_{N}(R) \subset \Delta \subset V$, and let $\Delta^{\prime}=\eta^{-1}(\Delta)$. Assume that $F$ and $G$ generate $A\left(\Delta^{\prime}\right)$ as a module over $\eta^{*} \mathscr{A}(\Delta)$. If $f$ is any element of $\mathscr{A}\left(\Delta^{\prime}\right)$, we can write $f=f_{e}+f_{0}$, $f_{e}, f_{0} \in \mathscr{A}\left(\Delta^{\prime}\right)$ where $f_{e}$ is even in the sense that it is constant on the fiber $\eta^{-1}(z), z \in \Delta$, and $f_{0}$ is odd in that if $\eta^{-1}(z)=\left(z^{\prime}, z^{\prime \prime}\right)$, then $f_{0}\left(z^{\prime}\right)=$ $-f_{0}\left(z^{\prime \prime}\right)$. To obtain such a decomposition, write $f_{e}(z)=\frac{1}{2}\left(f\left(z^{\prime}\right)+f\left(z^{\prime \prime}\right)\right)$ where $\eta^{-1}(\eta(z))=\left\{z^{\prime}, z^{\prime \prime}\right\}$, and define $f_{0}$ to be $f-f_{e}$. It is clear that $f_{e}$ is, in fact, even, and that $f_{0}$ is odd. It is easily verified that this decomposition of $f$ into a sum of even and odd parts is unique.

By hypothesis, if $h \in \mathscr{A}\left(\Delta^{\prime}\right)$, we have $h=f F+g G$ for some choice of $f, g \in \eta^{*} \mathscr{A}(\Delta)$. The functions $f$ and $g$ are both even, and it follows that if $h=h_{e}+h_{0}$, then

$$
h_{e}=f F_{0}+g G_{e}
$$

and

$$
h_{0}=f F_{0}+g G_{0} .
$$

By suitable choice of $h \in \mathscr{A}(\Delta)$, we can arrange that the pair $\left(h_{e}(z)\right.$, $\left.h_{0}(z)\right)$ be any point of $C^{2}$, so it follows that the determinant $\mathrm{D}(\mathfrak{z})=\left|\begin{array}{ll}F_{e}(z) & G_{e}(\mathfrak{z}) \\ F_{0}(z) & G_{0}(z)\end{array}\right|$ is zero for no choice of $z$.

However, $D$ is an odd function. Thus, continuing with the notation of Section I, $\triangleright \circ \circ \xi$ is a $C$-valued function on $S^{N}$ which is zero-free and odd in that if $p$ and $q$ are antipodal points in $S^{N}$, then $\triangleright \circ \xi(p)=$ $-\triangleright \circ \circ \xi(q)$. Since $C$ is topologically $R^{2}$ and since $N \geqq 2$, the BorsukUlam theorem implies that such an odd function has a zero, and the desired contradiction has been reached. 


\section{REFERENCES}

1. N. Alling, Extensions of meromorphic function rings over noncompact Riemann surfaces, I, Math. Zeit. 89 (1965), 273-299.

2. N. Bourbaki, Éléments de Mathématique, Algèbra Communtative, Hermann, 1961, Paris.

3. O. Forster, Some results on parallelizable Stein manifolds, Bull. Amer. Math. Soc. 73 (1967), 711-716.

4. O. Forster and K.J. Ramspott, Über die Darstellung analytischer Mengen, Sb. Bayer. Akad. Wiss., Math.-Nat. Kl. (1963), 89-99.

5. R. C. Gunning and H. Rossi, Analytic functions of several complex variables, Prentice-Hall, Inc., Englewood Cliffs, 1965.

6. P. Hilton and S. Wylie, Homology theory, Cambridge University Press, London and New York, 1960.

7. W. Rudin and E.L. Stout, Modules over polydisc algebras, Trans. Amer. Math. Soc. April 1969.

8. J.-P. Serre, Exposé XX Séminaire H. Cartan. 1951-1952. Reprinted by W.A. Benjamin, New York and Amsterdam, 1967.

Received June 13, 1968. The author is a Research Associate of the Office of Naval Research.

YALE UNIVERSITY 



\section{PACIFIC JOURNAL OF MATHEMATICS}

\section{EDITORS}

H. ROYDEN
Stanford University
Stanford, California

\section{R. R. PHELPS}

University of Washington

Seattle, Washington 98105
J. DUGUNDJI

Department of Mathematics

University of Southern California

Los Angeles, California 90007

\section{RICHARD ARENS}

University of California

Los Angeles, California 90024

\section{ASSOCIATE EDITORS}

E. F. BeCKenbaCh
B. H. NEUMANN

F. WOLF
K. YoshidA

\section{SUPPORTING INSTITUTIONS}

\author{
UNIVERSITY OF BRITISH COLUMBIA \\ CALIFORNIA INSTITUTE OF TECHNOLOGY \\ UNIVERSITY OF CALIFORNIA \\ MONTANA STATE UNIVERSITY \\ UNIVERSITY OF NEVADA \\ NEW MEXICO STATE UNIVERSITY \\ OREGON STATE UNIVERSITY \\ UNIVERSITY OF OREGON \\ OSAKA UNIVERSITY \\ UNIVERSITY OF SOUTHERN CALIFORNIA
}

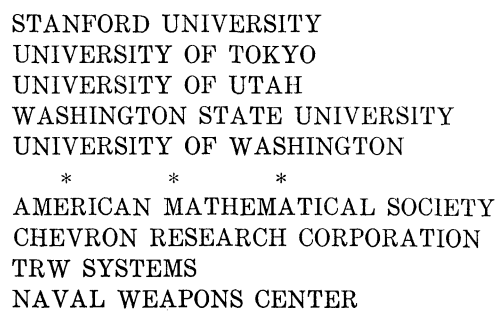

The Supporting Institutions listed above contribute to the cost of publication of this Journal, but they are not owners or publishers and have no responsibility for its content or policies.

Mathematical papers intended for publication in the Pacific Journal of Mathematics should be in typed form or offset-reproduced, double spaced with large margins. Underline Greek letters in red, German in green, and script in blue. The first paragraph or two must be capable of being used separately as a synopsis of the entire paper. It should not contain references to the bibliography. Manuscripts, in duplicate if possible, may be sent to any one of the four editors. Please classify according to the scheme of Math. Rev. 36, 1539-1546. All other communications to the editors should be addressed to the managing editor, Richard Arens, University of California, Los Angeles, California, 90024.

50 reprints are provided free for each article; additional copies may be obtained at cost in multiples of 50 .

The Pacific Journal of Mathematics is published monthly. Effective with Volume 16 the price per volume (3 numbers) is $\$ 8.00$; single issues, $\$ 3.00$. Special price for current issues to individual faculty members of supporting institutions and to individual members of the American Mathematical Society: $\$ 4.00$ per volume; single issues $\$ 1.50$. Back numbers are available.

Subscriptions, orders for back numbers, and changes of address should be sent to Pacific Journal of Mathematics, 103 Highland Boulevard, Berkeley, California, 94708.

PUBLISHED BY PACIFIC JOURNAL OF MATHEMATICS, A NON-PROFIT CORPORATION

Printed at Kokusai Bunken Insatsusha (International Academic Printing Co., Ltd.), 7-17, Fujimi 2-chome, Chiyoda-ku, Tokyo, Japan. 


\section{Pacific Journal of Mathematics \\ Vol. 29, No. $2 \quad$ June, 1969}

Bruce Langworthy Chalmers, On boundary behavior of the Bergman kernel function and related domain functionals ................... 243

William Eugene Coppage, Peirce decomposition in simple Lie-admissible power-associative rings .............................. 251

Edwin Duda, Compactness of mappings...................... 259

Earl F. Ecklund Jr., On prime divisors of the binomial coefficient......... 267

Don E. Edmondson, A modular topological lattice ............... 271

Phillip Alan Griffith, A note on a theorem of Hill ................... 279

Marcel Herzog, On finite groups with independent cyclic Sylow

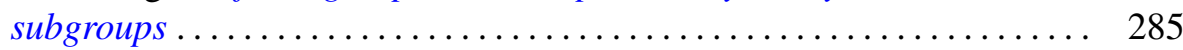

James A. Huckaba, Extensions of pseudo-valuations................. 295

S. A. Huq, Semivarieties and subfunctors of the identity functor ........ 303

I. Martin (Irving) Isaacs and Donald Steven Passman, Finite groups with small character degrees and large prime divisors. II ............ 311

Carl Kallina, A Green's function approach to perturbations of periodic

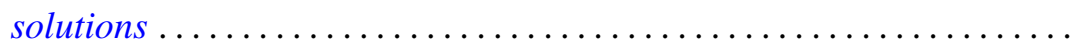

$\mathrm{Al}$ (Allen Frederick) Kelley, Jr., Analytic two-dimensional subcenter manifolds for systems with an integral ....................

Alistair H. Lachlan, Initial segments of one-one degrees ............ 351

Marion-Josephine Lim, Rank k Grassmann products ............. 367

Raymond J. McGivney and William Henry Ruckle, Multiplier algebras of

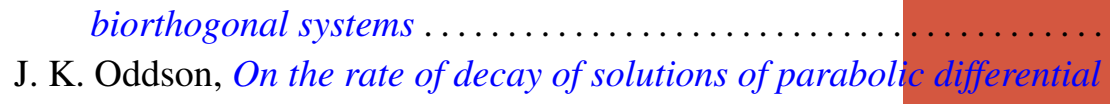

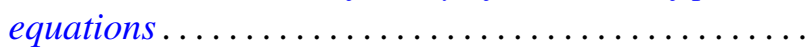

Helmut R. Salzmann, Geometries on surfaces ........... .

Annemarie Schlette, Artinian, almost abelian groups and their groups of automorphisms ............................

Edgar Lee Stout, Additional results on modules over polydisc algebras ...

Lajos Tamássy, A characteristic property of the sphere . .

Mark Lawrence Teply, Some aspects of Goldie's torsion theory. ...

Freddie Eugene Tidmore, Extremal structure of star-shaped sets ...

461

Leon Jarome Weill, Unconditional and shrinking bases in locally convex spaces... 